\author{
Part of Journal of Research of the National Bureau of Standards, Volume 28, \\ January 1942
}

\title{
EXPANSIVITY OF A VYCOR BRAND GLASS
}

\author{
By James B. Saunders
}

ABSTRACT

In a study of a new glass (96 percent silica, glass No. 790) used in heat-resisting glassware, its expansivity is compared with that of fused quartz. For this comparison a modification of Fizeau's method for determining very small differences in linear expansion was used and is presented. This method has the advantage of permitting the use of relatively long samp'es, the procurement of very sharp and well-defined interference bands, and the almost complete elimination of the effect of changes in the refractive index of air on the results.

The results indicate that the No. 790 glass has a coefficient of expansion approximately twice that of fused quartz at room temperature, an expansivity equal to fused quartz at approximately $300^{\circ} \mathrm{C}$., and an expansivity less than one-half of that for fused quartz, in the neighborhood of $700^{\circ} \mathrm{C}$.

A study of the physical properties of the new glass (96 percent silica, glass No. 790) used in heat-resisting glassware is of particular interest, since its manufacture demands a process ${ }^{1}$ quite different from that used in making most glasses. On heating this glass from room temperature to $900^{\circ} \mathrm{C}$., the expansivity is found to vary from approximately twice to less than one-half that of fused quartz. The proximity of these values to those of fused quartz and the exceptionally large percentage decrease in expansivity with increasing temperature are believed to be of general interest.

In the determination of expansivities of materials with such low expansion as fused quartz and glass No. 790, the usual methods do not permit of high precision. The usual interferometer method ${ }^{2}$ is ordinarily as good as any, but the accuracy of its results depends upon an accurate knowledge of the changes in the refractive index of air with temperature. The change in the refractive index of air requires a relatively large correction when low-expansion materials are being measured.

In this work a modification of Fizeau's differential interferometer method ${ }^{3}$ was used. That is, the three adjustable screws used by Fizeau to support the top plate were replaced by a fused-quartz tube. The length of this tube and that of the test specimen were chosen so that the resulting air correction was very small. Obviously this

\footnotetext{
1 Glass Industry 20, 269 (1939). See also U. S. Patents 2,106,744 and 2,150,694.

2 G. E. Merritt, BS, J. Research 10, 59 (1933) RP515. See also improvements by the author: J. Research NBS 23, 179 (1939) RP1227.

Ann. Phys, 128, 564 (1866).
} 
differential method also permits the use of specimens much longer than the usual interferometer method. It thus yields an accuracy in the difference between the expansivity of the sample and that of fused quartz which is believed to be much better than the accuracy of the accepted values for the expansivity of fused quartz. This particular modification of Fizeau's method may or may not be new.

Since the expansion of fused quartz is well known, a comparison of the expansion of No. 790 with that of fused quartz by the method described below will yield the value for No. 790 with an accuracy limited only by the accuracy with which that of fused quartz is known.

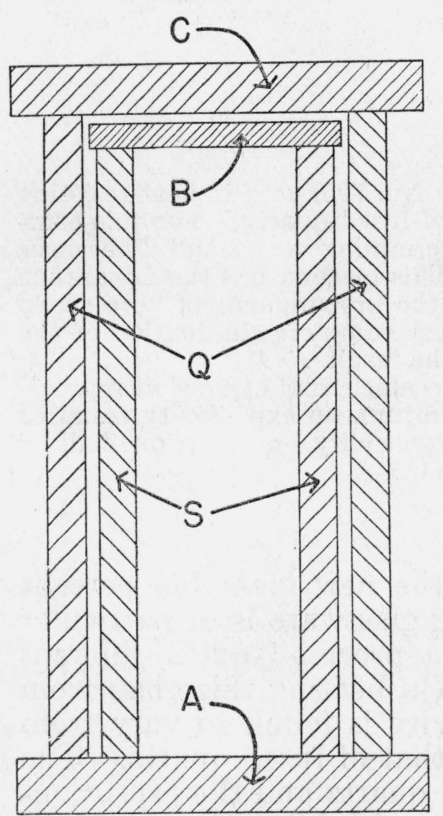

FIGURE 1.-Schematic drawing of the differential interferometer.

Figure 1 is a diagrammatic view of the interferometer arrangement used. The specimen of No. 790 is represented by $S$ and is in the form of a tube. A similar tube of fused quartz, having an inside diameter larger than the outside diameter of the No. 790 /specimen, is represented by $Q$. The plates $A, B$, and $C$ are made of fused quartz with plane surfaces. Plate $B$ is fine ground on the lower face and polished on its upper surface, the two surfaces being parallel. Plate $C$ is polished on both faces, which, however, are not quite parallel. The two ends of each tube are ground so that when they stand vertically on a horizontal surface, only three points, equally spaced, are in touches with the base plate and so that a plate resting on the tube (as $B$ on $S$, fig. 1 ) contacts the tube at only three equally spaced points. The lengths of the tubes are adjusted so as to have a very thin air space between plates $B$ and $C$. Temperature was determined by means of a thermocouple, the junction of which was located just below the center of the lower surface of plate $A$. The No. 790 specimen used was approximately $48 \mathrm{~mm}$ in length; the fused quartz tubing, $52 \mathrm{~mm}$ long; and the thickness of plate $B, 3 \mathrm{~mm}$. Much longer samples may be tested by this method, and the air space between plates $B$ and $C$ may be made less than $0.1 \mathrm{~mm}$ if the differential expansion permits.

When the various parts of the interferometer are properly adjusted, the two polished surfaces bounding the air space between plates $B$ and $C$ are nearly enough parallel to produce good broad interference bands when monochromatic light is used. The shortness of the light path between these two reflecting surfaces permits the procurement of sharp, brilliant bands such as are not obtainable in the usual method ${ }^{4}$ because the latter requires longer paths. The elimination of air-film errors was accomplished by overweighting one of the supports of each plate, $B$ and $C$, and applying the correction factor explained in a previous paper. ${ }^{5}$

4 See footnote 2 .

5 J. B. Saunders, J. Research NBS 23, 186 (1939) RP1227. 


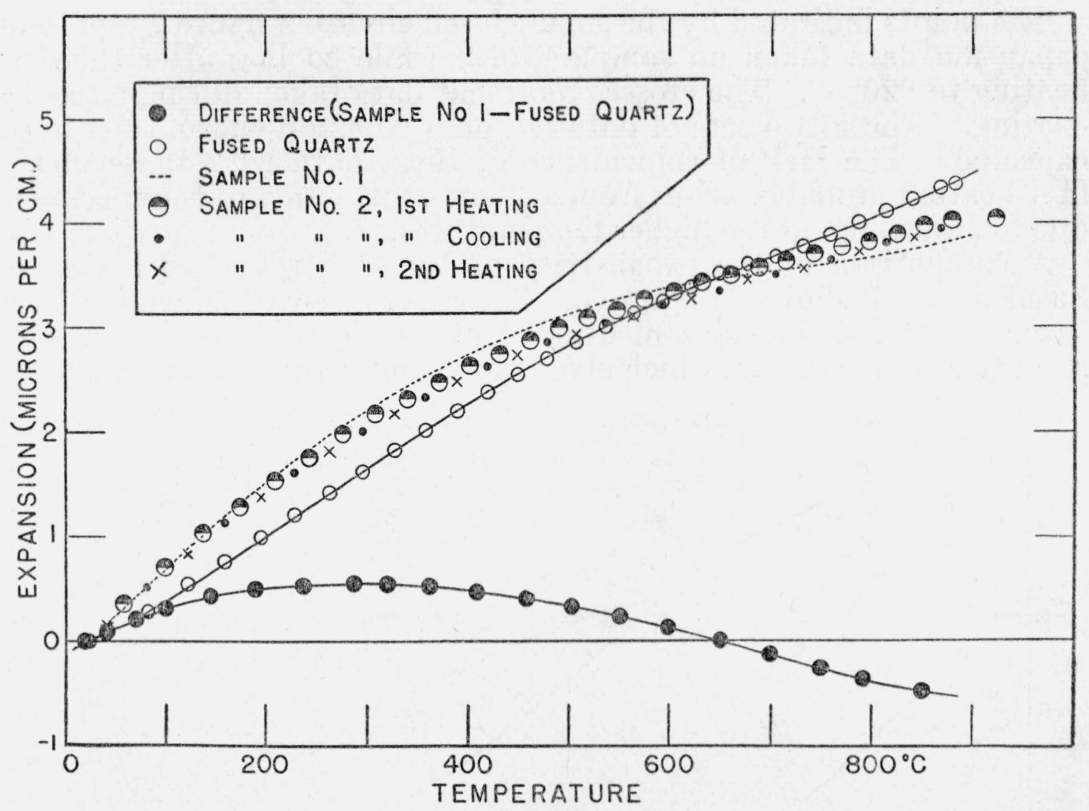

FigURE 2.-Expansion and differential expansion of fused quartz and Vycor (96 percent silica, glass No. 790).

The difference between the expansion of a specimen of No. 790 (represented as sample No. 1) and fused quartz was obtained by means of the above method, and the results are shown in figure 2 by the closed circles. An independent determination, using the usual method given in footnote 2 , was made on a sample of fused quartz which was taken from the same tube used in the above test. The results of this test are indicated by the open circles in figure 2. By applying the "Method of Averages," an empirical formula was found which fitted the data for the fused quartz within the errors of observation. The resultant formula is represented by the curve which passes through the open circles. A second formula was computed in the same manner to fit the differential-expansion data. By adding the corresponding ordinates representing the expansions obtained from these two formulas, a third formula representing the expansion of sample No. 1 was obtained. This third formula is represented in figure 2 by the dotted curve.

To check these results of the differential method, a second sample of Vycor No. 790 (sample No. 2) was used. The usual interferometer method was applied to obtain the expansion of this sample at various temperatures, and the results are indicated in figure 2 by the halfclosed circles. The discrepancy between the two samples of Vycor is not surprising, since the correction arising from the changes in the refractive index of air is large compared to the fringe shift when the usual interferometry method is applied in air. Also, the two samples could easily yield different values, since sample No. 1 was taken from a relatively thin-walled tube and sample No. 2 from a flask having much thicker walls. 
The points indicated by the small closed circles in figure 2 represent expansion data taken on sample No. 2 while cooling after the first heating to $920^{\circ} \mathrm{C}$. The crosses represent data taken during a second heating. These two sets of data fall on a common curve, as is to be expected. The lack of coincidence of these data with those of the first heating probably arises from a slight contraction of the specimen, due to annealing, at the higher temperatures.

A comparison of the expansivity of Vycor No. 790 with that of fused quartz is shown in figure 3. The curves were obtained from the three expansion formulas mentioned above, by differentiation with respect to temperature, which gives the slopes of the expansion curves.

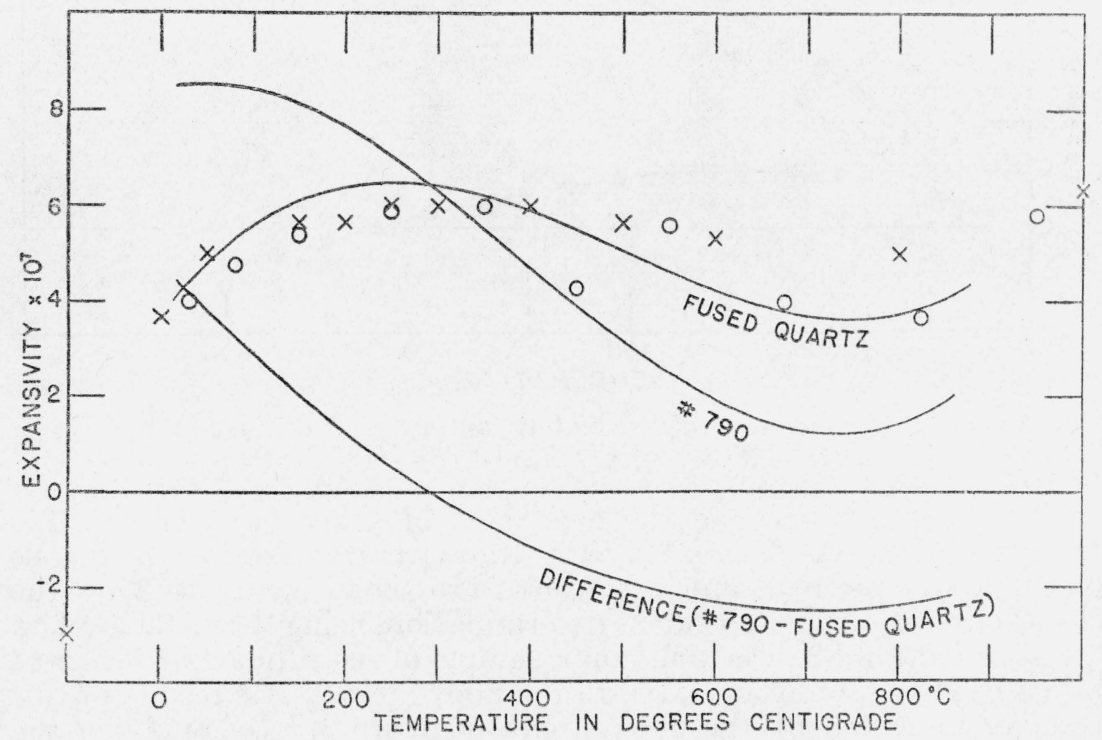

FIGURE 3.-Expansivity of fused quartz and Vycor (96 percent of silica, glass No. 790).

Crosses represent values selected by Sosman and circles represent values derived from Souder and Hidnert's data.

At room temperature the No. 790 glass has an expansivity approximately twice that of fused quartz, whereas at $300^{\circ} \mathrm{C}$ the two are nearly equal. At $700^{\circ} \mathrm{C}$ the expansivity of the sample of No. 790 is less than one-half that of fused quartz. Above $900^{\circ} \mathrm{C}$ the specimen either begins to deform under its own weight plus that of plate $B$ or it undergoes density changes which are independent of temperature variations. It may be mentioned that most glasses have expansivities which increase with increasing temperature, whereas the above samples of No. 790 have a decreasing expansivity from room temperature up to $700^{\circ} \mathrm{C}$ and an increasing expansivity from there on to $900^{\circ} \mathrm{C}$.

In order to eliminate errors arising from temperature gradients, each measurement was taken after temperature equilibrium had been well established. With the differential interferometer, the position of the two tubes (No. 790 and fused quartz) with respect to each 
other insured that the average temperature of each, from end to end, was practically the same. However, any error in the temperature measurement which is of the same magnitude at all temperatures introduces no error in the expansivity values. The air correction for refractive-index changes in the air is almost nil for the differential data, but large for the absolute determinations. The average error in the difference between the expansivity of fused quartz and No. 790 is believed to be less than 2 percent. Although this accuracy is not attained in the direct measurements on fused quartz, it is believed that the values reported here are as good as any of those reported by other observers.

A comparison of expansivity values found for the fused quartz used in this test with those reported by other observers would seem to be of interest. For this purpose a series of points (indicated by crosses in fig. 3) are given and represent values computed from volumetric values which were selected by Sosman. ${ }^{6}$ The results of work published by Souder and Hidnert ${ }^{7}$ of the National Bureau of Standards is represented by the circles shown in figure 3. These latter points represent the average expansivity over temperature ranges, of which the abscissas are the average temperatures of these ranges.

Washington, August 19, 1941.

- R. B. Sosman, The Properties of Silica, chapter XX, table XX.2, p. 362 (Chemical Catalog Co., Inc., New York, N. Y., 1927.

7 Sci. Pap. BS 21, 1 (1926) S524. 